\title{
Combination of bosentan with epoprostenol in pulmonary arterial hypertension: BREATHE-2
}

\author{
M. Humbert*, R.J. Barst", I.M. Robbins ${ }^{\Uparrow}$, R.N. Channick ${ }^{+}$, N. Galiè ${ }^{\S}$, A. Boonstra ${ }^{f}$, L.J. Rubin ${ }^{+}$, \\ E.M. Horn", A. Manes ${ }^{\S}$, G. Simonneau*
}

Combination of bosentan with epoprostenol in pulmonary arterial hypertension: BREATHE-2. M. Humbert, R.J. Barst, I.M. Robbins, R.N. Channick, N. Galiè, A. Boonstra, L.J. Rubin, E.M. Horn, A. Manes, G. Simonneau. (C) ERS Journals Ltd 2004.

ABSTRACT: The efficacy and safety of combining bosentan, an orally active dual endothelin receptor antagonist and epoprostenol, a continuously infused prostaglandin, in the treatment of pulmonary arterial hypertension (PAH) was investigated.

In this double-blind, placebo-controlled prospective study, 33 patients with PAH started epoprostenol treatment $\left(2 \mathrm{ng} \cdot \mathrm{kg}^{-1} \mathrm{~min}^{-1}\right.$ starting dose, up to $14 \pm 2 \mathrm{ng} \cdot \mathrm{kg}^{-1} \mathrm{~min}^{-1}$ at week 16) and were randomised for 16 weeks in a $2: 1$ ratio to bosentan $(62.5 \mathrm{mg}$ b.i.d for 4 weeks then $125 \mathrm{mg}$ b.i.d) or placebo.

Haemodynamics, exercise capacity and functional class improved in both groups at week 16. In the combination treatment group, there was a trend for a greater (although nonsignificant) improvement in all measured haemodynamic parameters. There were four withdrawals in the bosentan/epoprostenol group (two deaths due to cardiopulmonary failure, one clinical worsening, and one adverse event) and one withdrawal in the placebo/epoprostenol group (adverse event).

This study showed a trend but no statistical significance towards haemodynamics or clinical improvement due to the combination of bosentan and epoprostenol therapy in patients with pulmonary arterial hypertension. Several cases of early and late major complications were reported. Additional information is needed to evaluate the risk/benefit ratio of combined bosentan-epoprostenol therapy in pulmonary arterial hypertension.

Eur Respir J 2004; 24: 353-359.
*Hôpital Antoine Béclère, Université ParisSud, Clamart, France. ${ }^{\#}$ Columbia University College of Physicians and Surgeons, New York, NY, "Vanderbilt University School of Medicine, Nashville, TN, and ${ }^{+}$Division of Pulmonary and Critical Care Medicine, University of California at San Diego, La Jolla, CA, USA. ${ }^{\S}$ Università di Bologna, Bologna, Italy. ${ }^{f}$ Vrije Universiteit, Amsterdam, the Netherlands.

Correspondence: M. Humbert, Hôpital Antoine Béclère, Assistance Publique, Hôpitaux de Paris, Université Paris-Sud, 157 rue de la porte de Trivaux, 92140 Clamart, France. Fax: 33 146303824

E-mail: marc.humbert@abc.ap-hop-paris.fr

Keywords: Bosentan, endothelin receptors, epoprostenol, pulmonary arterial hypertension, scleroderma

Received: March 52004

Accepted after revision: June 12004

This study was supported by Actelion Pharmaceuticals Ltd, Allschwil, Switzerland.
Pulmonary arterial hypertension (PAH) is an uncommon disease characterised by a progressive increase in pulmonary vascular resistance leading to right ventricular failure and death [1]. PAH can be idiopathic (referred to as primary pulmonary hypertension-PPH), or occur as a complication of various conditions, including scleroderma [2] or systemic lupus erythematosus [3]. The pathogenesis of PAH involves multiple and complex mechanisms triggered by endothelial dysfunction in the pulmonary bed, resulting in pulmonary vasoconstriction and vascular remodelling. An imbalance between vasoconstrictor/vasodilator activities in favour of vasoconstriction could be responsible for altered pulmonary vascular tone and structure [4]. In PAH patients, relaxing factors such as prostacyclin [5] are decreased and nitric oxide synthesis is impaired [6], whereas constricting factors including thromboxane [7], serotonin [8], and endothelin [9] are increased. Restoration of this imbalance by targeted therapies such as prostacyclin and endothelin receptor antagonists should further improve treatment options for the management of PAH.

Prostacyclin (epoprostenol), a potent pulmonary vasodilator, decreases pulmonary vascular resistance and improves the survival of patients with severe PAH [10-15]. Despite major improvements in prognosis, mortality in patients with severe PAH treated with epoprostenol is still high, emphasising the

For editorial comments see page 339. need for novel therapeutic approaches in this patient population. In addition, epoprostenol is associated with dose-related side-effects (e.g. diarrhoea, flushing, headache, jaw pain, hypotension) caused by systemic vasodilation and carries the risk of line sepsis and rebound $\mathrm{PAH}$ from inadvertent interruption of infusion [10]. It has been recently demonstrated that predictors of survival in epoprostenol-treated patients include, among others, improvements in haemodynamic variables on epoprostenol. The present authors hypothesised that addition of bosentan, an orally active dual endothelin receptor antagonist [16-18], could further improve haemodynamics in patients initiated on intravenous epoprostenol, and by inference improve long-term results. Additional bosentan therapy may also reduce the need to up-titrate the epoprostenol dose and, therefore, potentially decrease epoprostenol dose-related side-effects.

The objectives of the Bosentan Randomized trial of Endothelin Antagonist Therapy for PAH (BREATHE-2) were to investigate the efficacy and safety of the combination of bosentan and epoprostenol in the treatment of patients with severe PAH.

\section{Patients and methods}

\section{Patient selection}

Enrolled patients had severe PAH in modified New York Heart Association (NYHA) functional classes [19] III or IV 
and were scheduled for epoprostenol therapy within 2 weeks of screening. PAH was either primary or associated with connective tissue disease. Patients were excluded if they had moderate to severe interstitial lung disease (i.e. total lung capacity $<60 \%$ or high resolution computed tomography scan total score $>2$ [20]), if they had started or stopped any PAH treatment within 1 month of screening, or were receiving glibenclamide (glyburide), cyclosporine-A, and/or tacrolimus.

The study was conducted according to the Helsinki Declaration of 1975 and amendments, in adherence to the International Conference of Harmonization Good Clinical Practice Guidelines and to the US Federal Register (1997). The protocol was approved by the local ethics review committees and written informed consent was obtained.

\section{Study design}

The study was a double-blind, randomised, placebocontrolled trial and was conducted in four centres in the USA and three centres in Europe (France, Italy, and the Netherlands) (fig. 1). A total of 33 enrolled patients started epoprostenol treatment $\left(2 \mathrm{ng} \cdot \mathrm{kg}^{-1} \mathrm{~min}^{-1}\right)$. Two days later they were randomised to receive bosentan or placebo (2:1 ratio) and another 2 days later the epoprostenol dose was increased to $4 \mathrm{ng} \cdot \mathrm{kg}^{-1} \mathrm{~min}^{-1}$. The subsequent maximum dose increase of epoprostenol was $2 \mathrm{ng} \cdot \mathrm{kg}^{-1} \mathrm{~min}^{-1}$ at 2-week intervals to reach a target dose of $12-16 \mathrm{ng} \cdot \mathrm{kg}^{-1} \mathrm{~min}^{-1}$ between week 14 and 16 . Upon randomisation, patients received either bosentan $62.5 \mathrm{mg}$ b.i.d for 4 weeks followed by the target dose $(125 \mathrm{mg}$ b.i.d) or placebo. The double-blind study duration was 16 weeks.

\section{Outcome measures}

Patients were evaluated after 1, 4, 6 (European centres only), 8, 12, and 16 weeks of therapy. The primary efficacy parameter was change from baseline to week 16 in total pulmonary resistance (TPR), determined by right heart

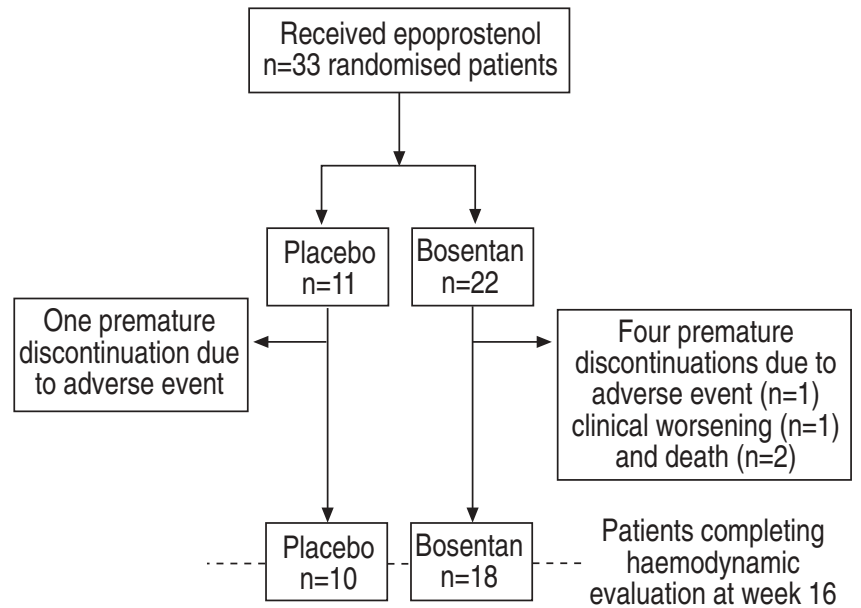

Fig. 1.-Study profile.

catheterisation. TPR is expected to provide prognostic information. A significant fall in TPR $(30 \%$ relative to baseline value) has been reported to be predictive of improved survival after 3 months of epoprostenol therapy in PPH patients [11]. Secondary efficacy parameters included the change in cardiac index (CI), pulmonary vascular resistance (PVR), mean pulmonary artery pressure (mPAP), and mean right atrial pressure (mRAP). Secondary measures of efficacy also included the 6-min walk distance [21], the dyspnoea-fatigue rating [22] and modified NYHA functional class of PAH [19]. Safety was assessed by adverse event recording, laboratory assessment and electrocardiogram.

\section{Statistical analysis}

A sample size of 20 patients on bosentan and 10 on placebo was required to detect a treatment difference in the mean

Table 1.-Demographic characteristics at baseline in the placebo/epoprostenol and bosentan/epoprostenol groups (intent-totreat population)

\begin{tabular}{lcc}
\hline Characteristic & Placebo/epoprostenol & Bosentan/epoprostenol \\
\hline Subjects n & 11 & 22 \\
M:F & $5(45): 6(55)$ & $5(23): 17(77)$ \\
Age yrs & $47 \pm 19(15-68)$ & $45 \pm 17(16-69)$ \\
Weight kg & $78 \pm 16(53-103)$ & $70 \pm 21(40-109)$ \\
Ethnic group & $10(91)$ & $18(82)$ \\
Caucasian/White & $1(9)$ & $1(5)$ \\
Black & & $1(5)$ \\
Asian & & $2(9)$ \\
Other & & $17(77)$ \\
Aetiology of PAH & $10(91)$ & $4(18)$ \\
Primary & $1(9)$ & $1(5)$ \\
Scleroderma & & $17(77)$ \\
Systemic lupus erythematosus & & $5(23)$ \\
Modified NYHA functional class & $8(73)$ & $10(45)$ \\
III & $3(27)$ & $19(86)$ \\
IV & $4(36)$ & $19(86)$ \\
Clinical signs of heart failure & & $14(64)$ \\
Concomitant PAH medications (only when $>4$ in at least one group) & $7(32)$ \\
Antithrombotic agents & $10(91)$ & $6(27)$ \\
High-ceiling diuretics & $10(91)$ & $6(27)$ \\
Potassium sparing agents & $5(45)$ & $13 \pm 30(1-138)$ \\
Cardiac glycosides & $2(18)$ & $3(27)$ \\
Calcium channel blockers & $4(36)$ & \\
Use of supplemental oxygen & $15 \pm 21(1-61)$ & \\
Time since diagnosis months & & \\
\hline
\end{tabular}

Data are presented as $\mathrm{n}(\%)$ of patients or mean $\pm \mathrm{SD}$ (range). M: male; F: female; PAH: pulmonary arterial hypertension; NYHA: New York Heart Association. 

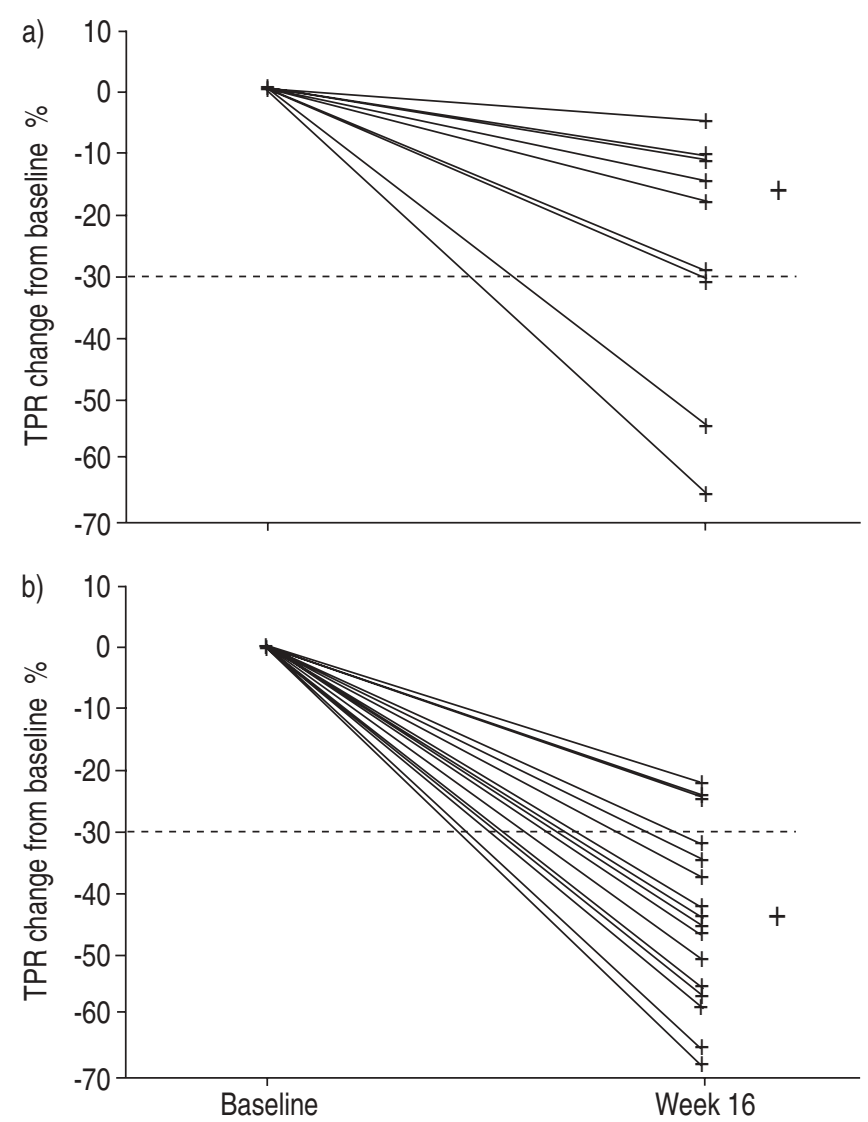

Fig. 2.- Total pulmonary resistance (TPR) change from baseline for patients on a) placebo/epoprostenol $(\mathrm{n}=10)$ and on b) bosentan/ epoprostenol $(\mathrm{n}=19)$. +: median values for each group at week 16 . Results presented are for patients who completed the 16-week study. The dashed line indicates a 30\% reduction in TPR.

percentage change in TPR of $28 \%(\alpha$-probability $=0.05$; type-II error $\beta=0.20,80 \%$ power). Patients who discontinued study medication due to an adverse event, lung transplantation or death were analysed using the assessment recorded at the time of premature withdrawal. In the event that no assessment was recorded, patients were assigned the worst rank value. All other patients without a week 16 assessment were assigned a zero change from baseline for haemodynamic parameters and had their last 6-min walk distance, dyspnoea-fatigue rating, and modified NYHA functional class carried forward.
The significance levels of the differences between treatment groups were evaluated with the two-samples Student's t-test and, additionally, the Mann-Whitney U-test. Data are expressed as median or mean \pm SEM and demographic data as mean \pm SD.

\section{Results}

A total of 33 patients were included in the study ( 22 patients received bosentan/epoprostenol and 11 received placebo/ epoprostenol) (fig. 1). One code break due to PAH worsening occurred before the week 16 assessment for a combined treatment patient who, thereafter, continued unblinded combined treatment.

\section{Demographics and baseline characteristics}

The study population was mostly composed of Caucasian PPH patients (table 1). All patients were in Class III $(76 \%)$ or IV $(24 \%)$ at baseline. Patients were taking at least one medication for PAH (mostly diuretics and anticoagulant agents). The bosentan/epoprostenol group included more women and more patients with scleroderma and clinical signs of heart failure.

\section{Cardiopulmonary haemodynamics}

TPR decreased from baseline to week 16 in both the bosentan/epoprostenol and the placebo/epoprostenol groups (fig. 2, table 2). The decrease in TPR was greater in the bosentan/epoprostenol group $(-36.3 \pm 4.3 \%$, mean \pm SEM) than in the placebo/epoprostenol group $(-22.6 \pm 6.2 \%)$, although the treatment group difference was not statistically significant ( $\mathrm{p}=0.08$ with Student's t-test and Mann-Whitney U-test) (table 2). Due to the substitution rules for missing data and the small number of patients, the median may be a better indication of the potential difference between treatment groups. A greater treatment difference was observed in the median change $\left(-648 \mathrm{dyn} \cdot \mathrm{s}^{-1} \mathrm{~cm}^{5}(-39.6 \%)\right.$ for bosentan/epoprostenol versus $-191 \mathrm{dyn} \cdot \mathrm{s}^{-1} \mathrm{~cm}^{5}(-14.3 \%)$ for placebo/epoprostenol).

Other haemodynamic parameters (CI, PVR, mPAP, and mRAP) improved from baseline in both treatment groups (table 2) and there were nonsignificant trends in favour of the bosentan/epoprostenol group.

\section{Exercise capacity}

Both treatment groups attained clinically relevant increases in the 6-min walk distance (68 $\mathrm{m}$ (median) in the

Table 2. - Haemodynamics for the placebo/epoprostenol and bosentan/epoprostenol groups at baseline and week 16 (intent-totreat population)

\begin{tabular}{|c|c|c|c|c|c|c|c|}
\hline \multirow[t]{2}{*}{ Haemodynamic parameter } & \multicolumn{3}{|c|}{ Placebo/epoprostenol } & \multicolumn{3}{|c|}{ Bosentan/epoprostenol } & \multirow[t]{2}{*}{ p-value } \\
\hline & Baseline & Week 16 & $\%$ change & Baseline & Week 16 & $\%$ change & \\
\hline TPR dyn $\cdot \mathrm{s}^{-1} \mathrm{~cm}^{5}$ & $1628 \pm 154$ & $1242 \pm 153$ & $-22.6 \pm 6.2$ & $1697 \pm 142$ & $1016 \pm 78$ & $-36.3 \pm 4.3$ & 0.08 \\
\hline $\mathrm{CI} \mathrm{L} \cdot \mathrm{min}^{-1} \mathrm{~m}^{2}$ & $1.7 \pm 0.2$ & $2.3 \pm 0.2$ & $37.9 \pm 13.3$ & $1.7 \pm 0.1$ & $2.5 \pm 0.1$ & $48.7 \pm 11.0$ & 0.6 \\
\hline $\operatorname{PVR}^{\top} \mathrm{dyn} \cdot \mathrm{s}^{-1} \mathrm{~cm}^{5}$ & $1426 \pm 140$ & $1050 \pm 154$ & $-25.7 \pm 7.2$ & $1511 \pm 129$ & $947 \pm 104$ & $-35.2 \pm 5.4$ & 0.3 \\
\hline mPAP mmHg & $60.9 \pm 2.9$ & $59.2 \pm 3.2$ & $-2.2 \pm 3.6$ & $59.2 \pm 4.0$ & $52.5 \pm 2.4$ & $-9.0 \pm 6.0$ & 0.3 \\
\hline mRAP mmHg & $11.9 \pm 2.2$ & $12.2 \pm 1.8$ & $0.3 \pm 1.3^{\#}$ & $11.9 \pm 1.1$ & $10.0 \pm 1.2$ & $-1.9 \pm 1.4^{\#}$ & 0.7 \\
\hline
\end{tabular}

Data are presented as mean \pm SEM. TPR: total pulmonary resistance; CI: cardiac index; PVR: pulmonary vascular resistance; mPAP: mean pulmonary artery pressure; mRAP: mean right atrial pressure. Note: the two bosentan patients who died and the two patients (one bosentan and one placebo) who were withdrawn were assigned the worst value observed at the 16 week time point or at withdrawal in all patients belonging to the same analysis population. \#: absolute change in mRAP; changes in mRAP are more meaningful when expressed as absolute changes rather than per cent changes because of the small absolute values and the large variation in the per cent changes due to the substitution rules; ${ }^{\top}$ : one placebo/epoprostenol and two bosentan/epoprostenol patients did not have a PVR calculation because of missing pulmonary artery wedge pressure. $\mathrm{n}=11$ (placebo/ epoprostenol) and 22 (bosentan/epoprostenol). 
bosentan/epoprostenol group versus $74 \mathrm{~m}$ (median) in the placebo/epoprostenol group) (fig. 3). The median dyspnoeafatigue ratings improved by 1.0 unit in the placebo/epoprostenol
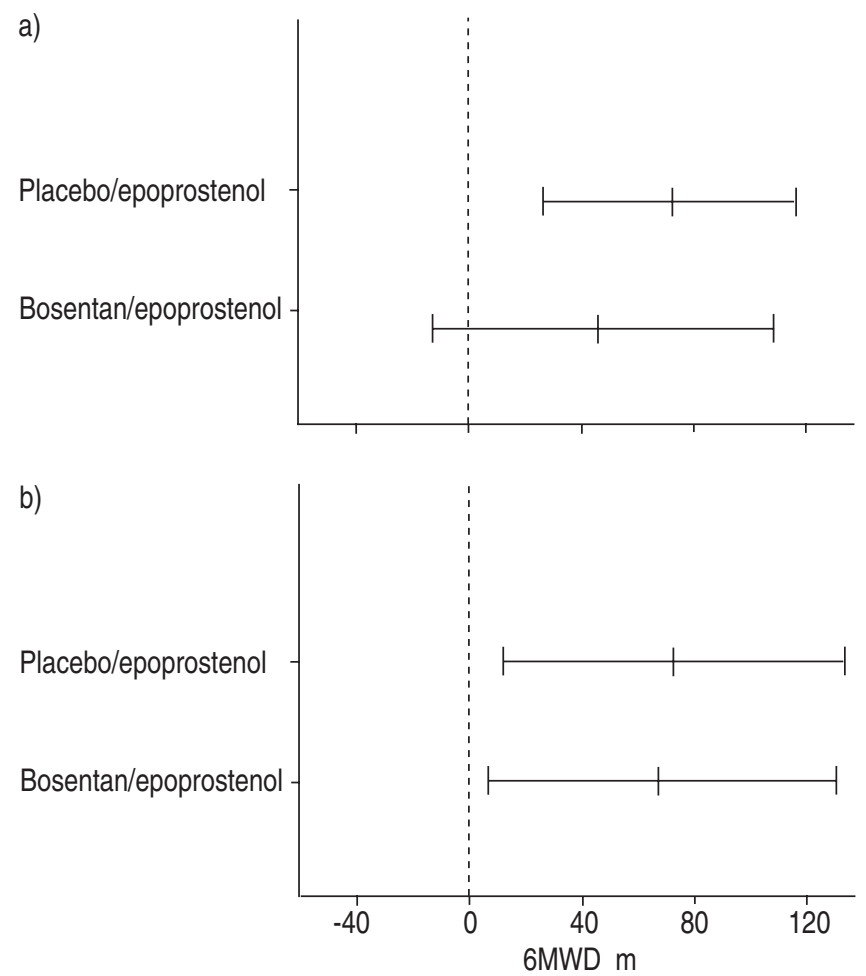

Fig. 3.-Six-minute walk distance (6MWD) for the placebo/ epoprostenol $(n=10)$ and bosentan/epoprostenol $(n=19)$ groups at a) baseline and b) week 16 (intent-to-treat population). Data are presented as a) mean $\pm 95 \% \mathrm{CI}$ and b) median $\pm 95 \% \mathrm{CI}$. Two patients on bosentan/epoprostenol therapy were assigned a $0-\mathrm{m}$ walk distance and a 0-dyspnoea-fatigue rating at week 16. Two patients (one on placebo/epoprostenol and one on bosentan/epoprostenol) were too impaired to walk and two patients on bosentan/epoprotenol did not perform the assessment. These patients were not included in the analysis.

Table 3. - Adverse events in the placebo/epoprostenol and bosentan/epoprostenol groups observed for $\geqslant$ three patients until 28 days after the end of the study (safety population)

\begin{tabular}{lcc}
\hline Adverse event & $\begin{array}{c}\text { Placebo/ } \\
\text { epoprostenol }\end{array}$ & $\begin{array}{c}\text { Bosentan/ } \\
\text { epoprostenol }\end{array}$ \\
\hline Subjects n & 11 & 22 \\
Pain in jaw & $10(91)$ & $13(59)$ \\
Diarrhoea & $3(27)$ & $12(55)$ \\
Flushing & $5(45)$ & $6(27)$ \\
Headache & $4(36)$ & $6(27)$ \\
Oedema lower limb & $1(9)$ & $6(27)$ \\
Limb pain & $2(18)$ & $5(23)$ \\
Nausea & $2(18)$ & $4(18)$ \\
Dermatitis & $1(9)$ & $4(18)$ \\
Cardiac failure & $2(18)$ & $3(14)$ \\
Upper respiratory tract infection & $1(9)$ & $3(14)$ \\
Abnormal hepatic function & $2(18)$ & $2(9)$ \\
Cough & $1(9)$ & $2(9)$ \\
Dizziness & $1(9)$ & $2(9)$ \\
Dyspnoea & $1(9)$ & $2(9)$ \\
Epistaxis & $1(9)$ & $2(9)$ \\
Myalgia & $1(9)$ & $2(9)$ \\
Worsening PHT & $2(18)$ & $1(5)$ \\
\hline
\end{tabular}

Data are presented as $\mathrm{n}(\%)$ of patients. PHT: worsening pulmonary hypertension. group and did not change in the bosentan/epoprostenol group. The treatment group differences for the walk test and dyspnoea-fatigue ratings were not statistically significant. These results were obtained after assigning a $0-\mathrm{m}$ walk distance and a 0 -dyspnoea-fatigue rating at week 16 to two patients on bosentan/epoprostenol therapy, which decreased the mean walk performance of the bosentan/epoprostenol group.

\section{Modified NYHA functional class}

Functional class of PAH improved from baseline to week 16 for 13 patients $(59 \%)$ in the bosentan/epoprostenol group and for five patients (45\%) in the placebo/epoprostenol groups (fig. 4). Among these improved patients, four bosentan/ epoprostenol patients and one placebo/epoprostenol patient were initially in Class IV. The treatment group difference was not statistically significant.

\section{Safety and tolerability}

The most frequently reported adverse events were those known to be associated with epoprostenol therapy (jaw pain, diarrhoea, flushing, and headache) (table 3). Except for diarrhoea, these adverse events were more frequent in the placebo/epoprostenol group. The only adverse event associated with bosentan therapy that occurred more frequently in patients treated with bosentan/epoprostenol than in those on epoprostenol alone was leg oedema ( $27 \%$ versus $9 \%)$. A clinically relevant decrease in haemoglobin concentration was observed in one patient receiving placebo/epoprostenol. Abnormal hepatic function (asymptomatic increases in the level of hepatic transaminases), which has been reported in previous trials with bosentan, was more frequent in the present study in the placebo/epoprostenol (18\%) group than in the bosentan/epoprostenol group ( $9 \%)$. Two patients, one in each treatment group, were withdrawn from the study because of increases in hepatic transaminases.

The number of serious adverse events associated with PAH (cardiopulmonary failure) was similar in the two treatments groups (14\% in the bosentan/epoprostenol group versus $18 \%$ in the placebo/epoprostenol group).

Two patients receiving bosentan/epoprostenol treatment

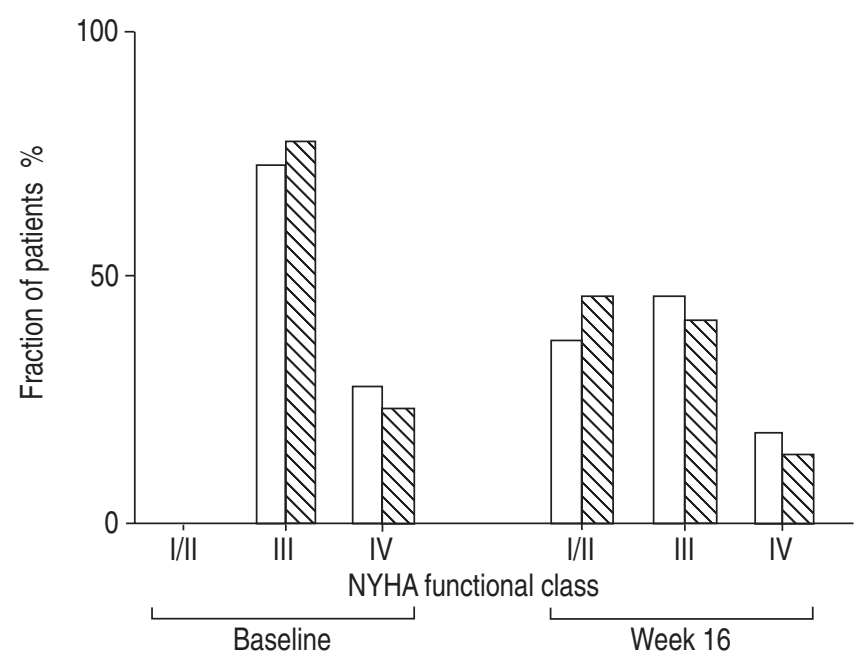

Fig. 4. - Modified New York Heart Association (NYHA) functional class for the placebo/epoprostenol $(\square)$ and bosentan/epoprostenol ( $\mathbb{\nabla}$ ) groups at baseline and week 16 (intent-to-treat population). 
Table 4.-Vital signs for the placebo/epoprostenol and bosentan/epoprostenol groups at baseline and week 16 (safety population)

\begin{tabular}{lrccrrrr}
\hline Vital signs & \multicolumn{3}{c}{ Placebo/epoprostenol } & & \multicolumn{2}{c}{ Bosentan/epoprostenol } \\
\cline { 2 - 3 } & Baseline & End of treatment & Absolute change & & Baseline & End of treatment & Absolute change \\
\hline Heart rate bpm & $85.2 \pm 4.5$ & $91.8 \pm 3.3$ & $6.6 \pm 3.6$ & & $83.8 \pm 2.1$ & $83.3 \pm 1.8$ & $-0.5 \pm 3.0$ \\
Systolic BP $\mathrm{mmHg}$ & $120.4 \pm 4.8$ & $110.5 \pm 4.9$ & $-9.8 \pm 3.3$ & & $110.2 \pm 2.7$ & $106.3 \pm 2.6$ & $-3.9 \pm 3.1$ \\
Diastolic BP mmHg & $75.9 \pm 4.6$ & $70.0 \pm 3.3$ & $-5.9 \pm 2.8$ & & $71.9 \pm 2.3$ & $65.9 \pm 2.3$ & $-6.0 \pm 2.7$ \\
\hline
\end{tabular}

Data are presented as mean \pm SEM. BP: blood pressure; bpm: beats per minute. $n=11$ (placebo/epoprostenol) and 22 (bosentan/epoprostenol).

died during the study; one class IV patient with PAH due to systemic sclerosis died from acute cardiopulmonary failure, the other patient (baseline class III PPH) became anaemic and subsequently developed pneumonia with rapidly progressing right heart failure. A third patient receiving bosentan/ epoprostenol treatment died after being withdrawn from the study for PAH worsening. These deaths were not considered related to study treatment by the clinical investigators, but rather reflected the severity and progressive nature of PAH.

Although the two groups received similar doses of epoprostenol, the haemodynamic improvement in the placebo/ epoprostenol group was associated with an increase in heart rate $(6.6 \pm 3.6$ mean \pm SEM), while in the bosentan/epoprostenol group a similar improvement was achieved with no increase in heart rate $(-0.5 \pm 3.0$ mean \pm SEM) (table 4$)$. Decreases in blood pressures were observed in both groups (table 4) although the decrease in systolic blood pressure was less in the bosentan/ epoprostenol group than in the placebo/epoprostenol group. Hypotension was reported for two patients both of whom were in the placebo/epoprostenol group.

\section{Discussion}

This is the first double-blind, randomised, placebo-controlled study combining an endothelin receptor antagonist with intravenous epoprostenol as initial therapy in NYHA class III and IV PAH patients. As expected from previous studies with epoprostenol [10-15] and with bosentan [16-18], both epoprostenol and combined bosentan/epoprostenol treatments improved haemodynamics, exercise capacity and functional class at week 16 in patients with severe PAH. The combination of bosentan and epoprostenol resulted in nonstatistically significant trends toward improvement in all haemodynamic variables compared to treatment with epoprostenol/placebo. In addition, no significant difference could be established between treatment groups in exercise capacity (walk distance and dyspnoea-fatigue rating) or NYHA functional class. The study was not powered to detect such changes. The absence of significant differences regarding primary and secondary endpoints could possibly be explained by the small sample size. Treatment efficacy may also have been lessened in the bosentan/epoprostenol group compared to the placebo/ epoprostenol group due to the presence of a larger percentage of scleroderma patients (18\% versus $9 \%$, respectively) for which morbidity and mortality remains quite high [23]. Previous studies have shown that, while bosentan improves the exercise capacity of PPH patients, it prevents the rapid deterioration of scleroderma patients [18]. Scleroderma patients have a worse prognosis than PPH patients even with similar baseline haemodynamics [24]. In the present study, one death and one withdrawal due to PAH worsening occurred in scleroderma patients, which may reflect the poor prognosis of these patients.

Substitution rules were applied for two patients who died on bosentan/epoprostenol during the study period, which resulted in large decreases in walk distance for these patients and a skewed distribution for the treatment group. The first patient died at day 15 during the initial titration period and did not receive full doses of either epoprostenol or bosentan. The second one died at day 111 after several complications including severe epistaxis, anaemia, and pneumonia. Analysis of these cases by the clinical investigators concluded that the deaths were not related to the combination treatment but rather reflected the severity and progressive nature of PAH.

The most frequent adverse events (jaw pain, diarrhoea, flushing, and headache) were those known to be associated with epoprostenol therapy $[10,12]$. The only adverse event associated with bosentan therapy that occurred more frequently in the combination therapy group was leg oedema, possibly related to a peripheral effect since the mean right atrial pressure tended to be reduced. Abnormal laboratory results including decreased haemoglobin concentration and elevated hepatic transaminases, have been reported in previous bosentan trials [16, 18]. Decreased haemoglobin concentration is not uncommonly observed with vasodilator therapy due to fluid retention resulting in haemodilution. The incidence of elevated hepatic transaminases was similar in the two groups ( $9 \%$ of bosentan/epoprostenol patients versus $18 \%$ in the placebo/epoprostenol group).

Interestingly, the combination therapy appeared to have a lower occurrence of the side-effects usually associated with epoprostenol (jaw pain, flushing, and headache). The combination therapy also induced less systemic hypotension than epoprostenol alone and had no significant effect on heart rate which, in contrast, increased with epoprostenol alone. These data suggest that bosentan may blunt activation of the sympathetic nervous system thereby reducing reflex tachycardia, a phenomenon that has also been observed with other neurohormonal antagonists such as ACE inhibitors [25] and $\beta$-blocking agents [26] and may require further investigation.

Pharmacokinetic interaction is unlikely to contribute to the profile of the combination therapy, since both drugs have different metabolic and excretion profiles $[27,28]$. In addition, a recent study in children with PAH reported no significant effect of epoprostenol on the pharmacokinetics of bosentan [29], although any potential effects of bosentan on epoprostenol concentrations were not studied.

Bosentan in combination with prostanoids may be of interest in clinical practice, as recently suggested by open series where, in contrast to the present study, bosentan was added to a long-standing prostanoid treatment. Consideration of adding bosentan when the efficacy of prostanoid therapy is deemed unsatisfactory requires further investigation. However, HOEPER et al. [30] have reported improvements of 6-min walk distance and peak oxygen consumption in patients with severe PAH treated with bosentan in addition to inhaled iloprost or oral beraprost. Additionally, an additive effect may allow the reduction of the dose of epoprostenol and hence its related side-effects. However, it remains to be seen if the benefit for the patient outweighs the increase in therapeutic cost. The main limitation of the current study is the small number of patients enrolled, which may have prevented 
statistically significant discrimination between the two treatment groups. In addition, a total of five patients were withdrawn from the study: there were four withdrawals in the bosentan/epoprostenol group (two deaths due to cardiopulmonary failure, one clinical worsening, one adverse event due to elevated levels of hepatic transaminases) and one withdrawal in the placebo/epoprostenol group (adverse event due to elevated levels of hepatic transaminases). The deaths and clinical worsening in the bosentan/epoprostenol group were attributed to the progression of the severe PAH. A definitive conclusion on the safety of the combination therapy could not be drawn.

The objective of the present study was to detect potential additive effects of the combination of bosentan and epoprostenol treatments. Therefore, simultaneous initiation of bosentan and epoprostenol along with controlled uptitration of both drugs was attempted for all patients. Alternatively, one treatment could have been added after stabilisation with the previous therapy. However, starting bosentan treatment for patients on stable epoprostenol therapy could have led to the enrolment of patients under a wide range of epoprostenol doses since no standardised treatment dosage is recognised.

In conclusion, the combination of bosentan and epoprostenol therapies may be a therapeutic option for the management of patients with severe pulmonary arterial hypertension. Although the findings from this randomised, placebocontrolled study indicate a nonsignificant trend for a greater improvement in haemodynamic parameters in favour of the combined therapy, the results should be interpreted with caution. Power was the major limitation of this study, which enrolled only 33 patients. Larger trials designed to address long-term efficacy and safety of bosentan plus epoprostenol in appropriately selected patients are required to confirm the value of this novel therapeutic strategy.

The following individuals have substantially contributed to the design and interpretation of the results of the study and are also authors of this manuscript: V.V. McLaughlin, A. Frost, D.B. Badesch.

Acknowledgements. The authors would like to acknowledge the collaboration and commitment of all the local investigators and their staff, without whom the present study would not have been possible. In particular, the authors would like to thank O. Sitbon from Hôpital Antoine Béclère, Clamart, France; E.B. Rosenzweig, K. Schmitt and A. Widlitz from Columbia University College of Physicians and Surgeons, New York, NY; A. Delmotte from Vanderbilt University School of Medicine, Nashville, TN; P. Summerville from the Rush Heart Institute, Center for Pulmonary Hypertension, Chicago, IL, USA; G. Boggian from Università di Bologna, Bologna, Italy.

\section{References}

1. Rubin LJ. Primary pulmonary hypertension. $N$ Engl $J$ Med 1997; 336: 111-117.

2. Stupi AM, Steen VD, Owens GR, Barnes EL, Rodnan GP, Medsger TA, Jr. Pulmonary hypertension in the CREST syndrome variant of systemic sclerosis. Arthritis Rheum 1986; 29: $515-524$

3. Robbins IM, Gaine SP, Schilz R, Tapson VF, Rubin LJ, Loyd JE. Epoprostenol for treatment of pulmonary hypertension in patients with systemic lupus erythematosus. Chest 2000; 117: 14-18.
4. Chen YF, Oparil S. Endothelial dysfunction in the pulmonary vascular bed. Am J Med Sci 2000; 320: 223-232.

5. Tuder RM, Cool CD, Geraci MW, et al. Prostacyclin synthase expression is decreased in lungs from patients with severe pulmonary hypertension. Am J Respir Crit Care Med 1999; 159: 1925-1932.

6. Giaid A, Saleh D. Reduced expression of endothelial nitric oxide synthase in the lungs of patients with pulmonary hypertension. N Engl J Med 1995; 333: 214-221.

7. Christman BW, McPherson CD, Newman JH, et al. An imbalance between the excretion of thromboxane and prostacyclin metabolites in pulmonary hypertension. $N$ Engl J Med 1992; 327: 70-75.

8. Herve P, Launay JM, Scrobohaci ML, et al. Increased plasma serotonin in primary pulmonary hypertension. $\mathrm{Am}$ J Med 1995; 99: 249-254.

9. Galiè N, Grigioni F, Bacchi-Reggiani L, et al. Relation of endothelin-1 to survival in patients with primary pulmonary hypertension. Eur J Clin Invest 1996; 26: Suppl. 1, 273.

10. Barst RJ, Rubin LJ, Long WA, et al. A comparison of continuous intravenous epoprostenol (prostacyclin) with conventional therapy for primary pulmonary hypertension. The Primary Pulmonary Hypertension Study Group. $N$ Engl J Med 1996; 334: 296-302.

11. Sitbon O, Humbert $\mathrm{M}$, Nunes $\mathrm{H}$, et al. Long-term intravenous epoprostenol infusion in primary pulmonary hypertension: prognostic factors and survival. $\mathrm{J} \mathrm{Am} \mathrm{Coll}$ Cardiol 2002; 40: 780-788.

12. Badesch DB, Tapson VF, McGoon MD, et al. Continuous intravenous epoprostenol for pulmonary hypertension due to the scleroderma spectrum of disease. A randomized, controlled trial. Ann Intern Med 2000; 132: 425-434.

13. Humbert M, Sanchez O, Fartoukh M, et al. Short-term and long-term epoprostenol (prostacyclin) therapy in pulmonary hypertension secondary to connective tissue diseases: results of a pilot study. Eur Respir J 1999; 13: 1351-1356.

14. Shapiro SM, Oudiz RJ, Cao T, et al. Primary pulmonary hypertension: improved long-term effects and survival with continuous intravenous epoprostenol infusion. $\mathrm{J}$ Am Coll Cardiol 1997; 30: 343-349.

15. McLaughlin VV, Shillington A, Rich S. Survival in primary pulmonary hypertension: the impact of epoprostenol therapy. Circulation 2002; 106: 1477-1482.

16. Channick RN, Simonneau G, Sitbon O, et al. Effects of the dual endothelin-receptor antagonist bosentan in patients with pulmonary hypertension: a randomised placebocontrolled study. Lancet 2001; 358: 1119-1123.

17. Badesch DB, Bodin F, Channick RN, et al. Complete results of the first randomized placebo-controlled study of bosentan, a dual endothelin receptor antagonist, in pulmonary hypertension. Curr Ther Res 2002; 63: 227-246.

18. Rubin LJ, Badesch DB, Barst RJ, et al. Bosentan therapy for pulmonary arterial hypertension. N Engl J Med 2002; 346: 896-903.

19. Recommendations on the management of pulmonary hypertension in clinical practice. Heart 2001; 86: Suppl. 1, 1-13.

20. Wells AU, Hansell DM, Rubens MB, et al. Fibrosing alveolitis in systemic sclerosis: indices of lung function in relation to extent of disease on computed tomography. Arthritis Rheum 1997; 40: 1229-1236.

21. Guyatt GH, Sullivan MJ, Thompson PJ, et al. The 6-minute walk: a new measure of exercise capacity in patients with chronic heart failure. Can Med Assoc J 1985; 132: 919923.

22. Feinstein AR, Fisher MB, Pigeon JG. Changes in dyspneafatigue ratings as indicators of quality of life in the treatment of congestive heart failure. Am J Cardiol 1989; 64: $50-55$

23. Stone JH, Wigley FM. Management of systemic sclerosis: the art and science. Semin Cutan Med Surg 1998; 17: 5564.

24. Kawut SM, Taichman DB, Archer-Chicko CL, Palevsky HI, 
Kimmel SE. Hemodynamics and survival in patients with pulmonary arterial hypertension related to systemic sclerosis. Chest 2003; 123: 344-350.

25. Jakobsen J, Glaus L, Graf P, et al. Unmasking of the hypotensive effect of nifedipine in normotensives by addition of the angiotensin converting enzyme inhibitor benazepril. J Hypertens 1992; 10: 1045-1051.

26. Packer M, Bristow MR, Cohn JN, et al. The effect of carvedilol on morbidity and mortality in patients with chronic heart failure. U.S. Carvedilol Heart Failure Study Group. N Engl J Med 1996; 334: 1349-1355.

27. Weber C, Gasser R, Hopfgartner G. Absorption, excretion, and metabolism of the endothelin receptor antagonist bosentan in healthy male subjects. Drug Metab Dispos 1999; 27: 810-815.

28. Brash AR, Jackson EK, Saggese CA, Lawson JA, Oates JA, FitzGerald GA. Metabolic disposition of prostacyclin in humans. J Pharmacol Exp Ther 1983; 226: 78-87.

29. Barst RJ, Ivy D, Dingemanse J, et al. Pharmacokinetics, safety, and efficacy of bosentan in pediatric patients with pulmonary arterial hypertension. Clin Pharmacol Ther 2003; 73: $372-382$

30. Hoeper MM, Taha N, Bekjarova A, Gatzke R, Spiekerkoetter E. Bosentan treatment in patients with primary pulmonary hypertension receiving nonparenteral prostanoids. Eur Respir $J$ 2003; 22: 330-334. 